\title{
FRAÇÕES DA MATÉRIA ORGÂNICA EM SOLOS SOB FORMAÇÕES DECIDUAIS NO NORTE DE MINAS GERAIS ${ }^{1}$
}

\author{
CAROLINA MALALA MARTINS ${ }^{2 *}$, LIOVANDO MARCIANO DA COSTA ${ }^{3}$, CARLOS ERNESTO GONÇALVES \\ REYNAUD SCHAEFER ${ }^{3}$, EMANUELLE MERCÊS BARROS SOARES ${ }^{3}$, SARA RAMOS DOS SANTOS ${ }^{3}$
}

\begin{abstract}
RESUMO - A dinâmica da matéria orgânica influencia os principais processos químicos, físicos e biológicos nos solos e determina muitas vezes seu comportamento químico e sua fertilidade. É fundamental a quantificação das frações que compõem a matéria orgânica para a compreensão dos processos pedogenéticos que refletem ou induzem as propriedades dos solos. O objetivo do estudo foi avaliar o comportamento da matéria orgânica do solo de diferentes perfis de solos sob formações deciduais, por meio do fracionamento químico da matéria orgânica e obtenção de suas diferentes frações oxidáveis. Dez perfis de solo foram descritos e coletados em áreas de formações deciduais em municípios de Minas Gerais e Bahia. Foram realizadas análises químicas e físicas em cada horizonte. Para as frações da matéria orgânica foi realizado o desmembramento de substâncias húmicas e posterior determinação de carbono de cada fração, quais sejam, humina (C-HUM), ácido fúlvico (CFAF) e ácido húmico (C-FAH), e calculadas suas relações e o percentual de cada fração em relação a $\mathrm{C}$ orgânico total (COT), \%FAF, \%FAH, \%HUM e \%EA, além das frações de C oxidável em cada horizonte avaliado. As substâncias húmicas que compõem a matéria orgânica dos solos de perfis de Florestas Estacionais Deciduais mostraram que este compartimento da MOS apresentou predomínio da fração humina, seguido dos ácidos húmicos e com menor teor de $\mathrm{C}$, e os ácidos fúlvicos para a maioria dos solos. Já as diferentes frações de C oxidável puderam apontar frações mais lábeis associadas aos horizontes superficiais e mais recalcitrantes aos horizontes subsuperficiais.
\end{abstract}

Palavras-chave: Semiárido. Matas Secas. Carbono orgânico do solo.

\section{FRACTION OF ORGANIC MATTER IN SOIL UNDER DECIDUOUS FORMATIONS IN THE NORTH OF MINAS}

\begin{abstract}
The dynamics of organic matter influences the main chemical, physical and biological processes in soil, and often determines their chemical behavior and fertility. Therefore, it is essential to quantify the fractions that make up the organic matter to the understanding of pedogenic processes that reflect or induce soil properties. The aim of the study was to evaluate the behavior of soil organic matter in different soil profiles under deciduous formations, through fractionation of organic matter and obtain their different oxidizable fractions. Ten soil profiles were described and collected in areas of deciduous formations in the state of Minas Gerais and Bahia. Chemical and physical analyzes were performed on each horizon. For organic matter fractions, the fractionation of humic substances and subsequent determination of carbon in each fraction was performed: humin (HUM - C), fulvic acid (C - FAF) and humic acid (C - FAH) and calculated their relations and percentage of each fraction relative to total organic carbon (COT) , \%FAF, \%FAH, \%HUM and \%EA, beyond fractions oxidizable $\mathrm{C}$ in each evaluated horizon. Humic substances that make up the organic matter in the soil of seasonal dry forests profiles showed that this compartment MOS showed a predominance of the humin fraction, followed by humic acids and lower content of $\mathrm{C}$, fulvic acids for most soils. However, the different fractions of oxidizable $\mathrm{C}$ could already point more labile fractions associated with surface horizons, and more recalcitrant to subsurface horizons.
\end{abstract}

Keywords: Semiarid. Dry forests. Organic carbon of soil.

\footnotetext{
*Autor para correspondência

${ }^{1}$ Recebido para publicação em 31/03/2014; aceito em 30/07/2015.

Parte da Tese de Doutorado da primeira autora no Programa de Pós-Graduação em Solos e Nutrição de Plantas pela Universidade Federal de Viçosa.

${ }^{2}$ Departamento de Ciências Ambientais e Tecnológicas, UFERSA, CEP 59625-900, Mossoró (RN); carolmalala@ufersa.edu.br.

${ }^{3}$ Departamento de Solos, UFV, CEP 36570-900, Viçosa (MG); liovandomc@yahoo.com.br, carlos.schaefer@ufv.br, emanuelle.soares@ufv.br, sarasantos.agro@gmail.com.
} 


\section{INTRODUÇ̃̃̃O}

As formações deciduais, conhecidas como Matas Secas, estão associadas aos mais diversos biomas brasileiros, havendo registro de sua ocorrência em formações de Cerrado, Caatinga, das regiões Centro-Oeste e Nordeste e também em formações florestais sempre verdes da floresta Amazônica, na região Norte, e da Atlântica, na região Sul do país (SCARIOT; SEVILHA, 2005). Apesar das variações que dificultam a identificação das Matas Secas o solo pode ser fator importante na comparação de diferentes fragmentos florestais, principalmente devido a características que influenciam na disponibilidade de água e nutrientes para a vegetação (SIQUEIRA et al., 2009).

Segundo Prado e Gibbs (1993), a formação Floresta Estacional Decidual deve representar um remanescente da floresta contínua, a qual foi interligada à Caatinga do Nordeste e aos chacos argentinos em um período seco do Pleistoceno. Nos últimos dois séculos estas florestas foram seriamente reduzidas a pequenos fragmentos e estão severamente perturbadas pela retirada indiscriminada da madeira, pela pecuária extensiva e pelo fogo (Werneck et al., 2000). Além da problemática referente à caracterização as Matas Secas sofrem constantes ameaças devido ao contínuo desmatamento decorrente da expansão das fronteiras agrícolas, que após o uso intensivo do solo substituem o cultivo por pastagens seguido pelo abandono da área (ESPÍRITO-SANTO et al., 2006).

Os estudos relacionados à matéria orgânica do solo (MOS) nas regiões de Matas Secas do Brasil são geralmente referentes à mineralização da serapilheira como fonte de nutrientes para as florestas. Assim, estudos que forneçam informações sobre as substâncias húmicas são importantes para a avaliação do ambiente edáfico porque podem contribuir para a compreensão da pedogênese e dos impactos do manejo do solo (Miranda et al., 2007). Além do fracionamento químico da MOS, identificar diferentes frações em termos de estabilidade pode ser outra forma de avaliação de seu comportamento. A determinação de frações de carbono oxidáveis em solos sob Matas Secas pode ser um interessante indicador da recalcitrância dos compostos orgânicos presentes e uma resposta a sua estabilidade em diferentes tipos de solos comuns ao respectivo ecossistema. Nessa análise, o carbono do solo é dividido em quatro frações (F1, F2, F3 e F4) que possuem diferentes graus de recalcitrância, trazendo informações referentes à labilidade do carbono orgânico do solo, fundamentais para os estudos, visando compreender sua perda e consequente compartimentalização no solo. As frações F1 e F2 são mais lábeis, estão associadas com a disponibilidade de nutrientes e a formação de macroagregados. Já as frações F3 e F4 são mais recalcitrantes, sendo que a F4 constitui "compartimento passivo" da matéria orgânica do solo (Chan et al., 2001).

Posto isso, o presente trabalho teve como objetivo avaliar a matéria orgânica do solo de diferentes tipos de solo sob Floresta Estacional Decidual, por meio do fracionamento químico da matéria orgânica e obtenção de diferentes frações oxidáveis do carbono orgânico.

\section{MATERIAL E MÉTODOS}

O trabalho foi realizado em municípios do Norte de Minas Gerais (Capitão Enéas, Porteirinha e Mato Verde) e Sudoeste da Bahia (Candiba) por se tratar de uma região de ecótono, haja vista o encontro dos biomas Caatinga, Cerrado e Mata Atlântica (IBGE, 2004). Dez perfis foram descritos e coletados em áreas de Floresta Estacional Decidual (Tabela 1), de acordo com a descrição morfológica e coleta sugeridas por Santos et al. (2013). A região apresenta característica de clima quente e seco típico da Caatinga e semiárido, e a temperatura se mantém relativamente alta ao longo do ano (Figura 2). De acordo com a classificação de Köppen-Geiger, o clima classifica-se como tropical de savana, inverno seco e verão chuvoso (SNLCS, 1979).

As amostras dos horizontes de cada perfil foram coletadas, secas ao ar, destorroadas e passadas em peneira de $2 \mathrm{~mm}$ de abertura de malha, obtendose a terra fina seca ao ar (TFSA) no período de junho e julho de 2010. Foram realizadas análises de $\mathrm{pH}$, $\mathrm{Ca}, \mathrm{Mg}$ e $\mathrm{K}$ trocáveis, $\mathrm{P}$ disponível e granulometria em triplicata, de acordo com métodos descritos em Embrapa (1997). A determinação do carbono orgânico total (COT) foi realizada através da oxidação utilizando solução de dicromato de potássio $0,167 \mathrm{~mol} \mathrm{~L}^{-1}$ e ácido sulfúrico concentrado, com aquecimento em bloco digestor (YEOMANS; BREMNER, 1988). Para a realização do fracionamento das substâncias húmicas foi selecionado um horizonte superficial e subsuperficial de cada perfil de solo coletado e utilizada a metodologia que se baseia na solubilidade em meio alcalino ou ácido e posterior determinação de carbono de cada fração, quais sejam, humina (C-HUM), ácido fúlvico (CFAF) e ácido húmico (C-FAH), método adaptado de Swift (1996) por Mendonça e Matos (2005). Foram calculadas as relações C-FAH/C-FAF, C-EA/CHUM e C-EA/COT, sendo EA o extrato alcalino (C$\mathrm{EA}=\mathrm{CFAF}+\mathrm{C}-\mathrm{FAH})($ Benites et al., 2003) e o percentual de cada fração em relação a $\mathrm{C}$ orgânico total (COT), \%FAF, \%FAH, \%HUM e \%EA. 

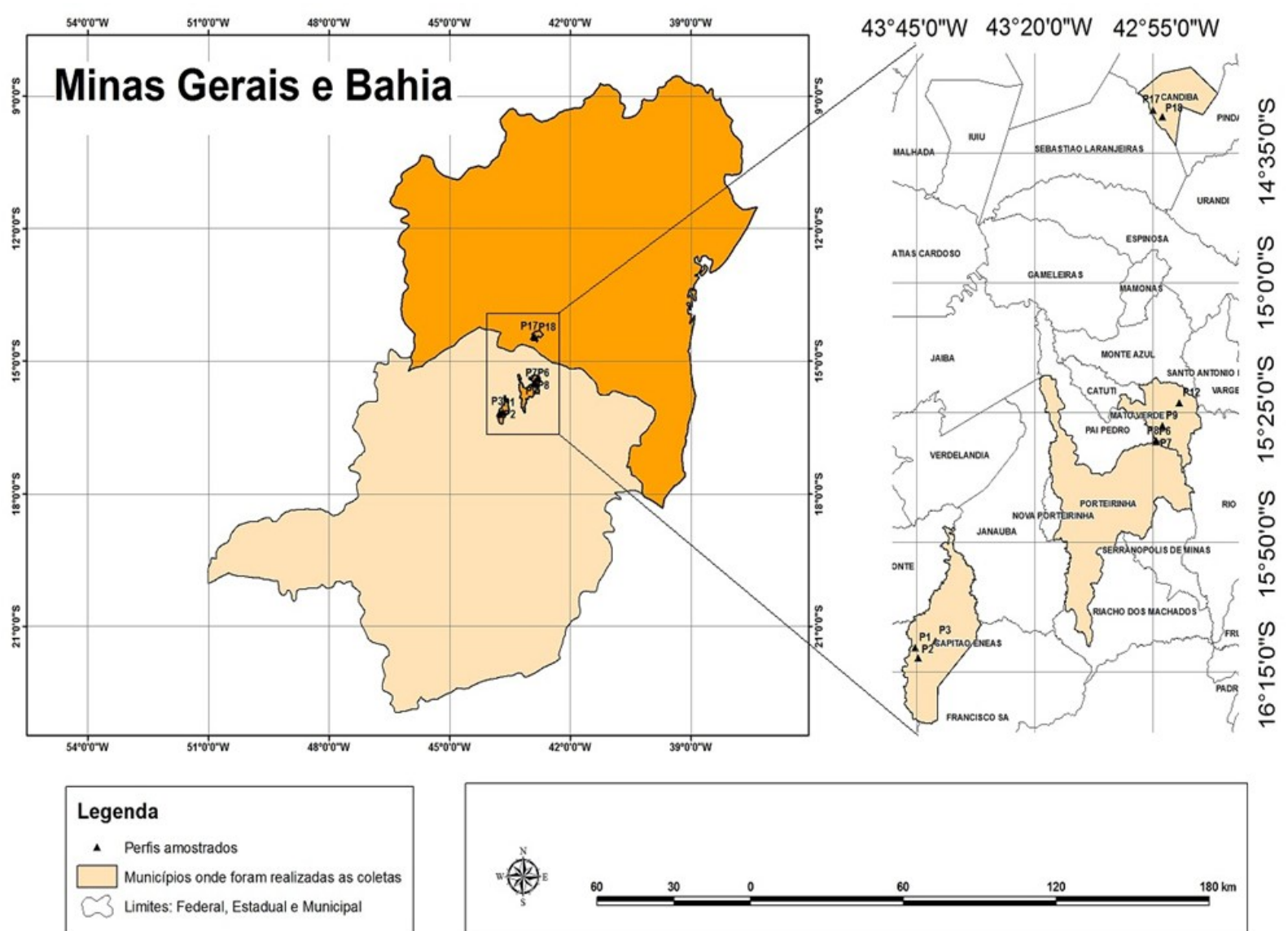

Figura 1. Localização das áreas estudadas e perfis coletados.

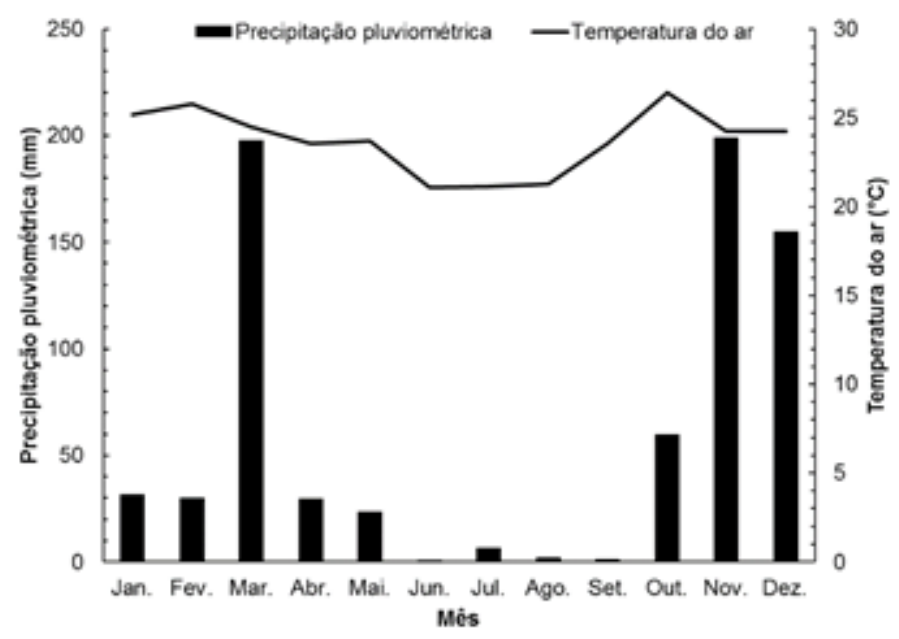

Figura 2. Precipitação e temperatura média mensal da região estudada no período da coleta, ano 2010. (Fonte: INMET, 2015). 
C. M. MARTINS et al.

Tabela 1. Classes de solos descritas e coletadas em áreas de formações deciduais

\begin{tabular}{lcl}
\hline Perfil & Localização & Classe \\
\hline P1 - NVe & Capitão Enéas-MG & NITOSSOLO VERMELHO Eutrófico típico \\
P2 - LVe & Capitão Enéas-MG & LATOSSOLO VERMELHO Eutrófico típico \\
P3 - CXbe & Capitão Enéas-MG & CAMBISSOLO HÁPLICO Tb Eutrófico típico \\
P4 - RLe & Porteirinha-MG & NEOSSOLO LITÓLICO Eutrófico típico \\
P5 - CXve & Porteirinha-MG & CAMBISSOLO HÁPLICO Ta Eutrófico típico \\
P6 - CXve & Mato Verde-MG & CAMBISSOLO HÁPLICO TaEutrófico \\
P7 - LVe & Mato Verde-MG & LATOSSOLO VERMELHO Eutrófico Típico \\
P8-CXbd & Mato Verde-MG & CAMBISSOLO HÁPLICO Tb distrófico típico \\
P9-CXbe & Candiba-BA & CAMBISSOLO HÁPLICO Tb Eutrófico típico \\
P10 - LVe & Candiba-BA & LATOSSOLO VERMELHO Eutrófico cambissólico \\
\hline
\end{tabular}

O teor de carbono orgânico determinado em diferentes graus de oxidação foram obtidos pelo método proposto por Mendonça e Matos (2005), adaptado de Chan et al. (2001), em que o solo é oxidado por uma solução de $\mathrm{K}_{2} \mathrm{Cr}_{2} \mathrm{O}_{7} 0,167 \mathrm{~mol} \mathrm{~L}^{-1}$ em meio ácido em três diferentes concentrações de $\mathrm{H}_{2} \mathrm{SO}_{4}(3$, 6 e $9 \mathrm{~mol} \mathrm{~L}^{-1}$ ), obtendo-se quatro frações: Fração 1 (F1) - C oxidado $\mathrm{K}_{2} \mathrm{Cr}_{2} \mathrm{O}_{7}$ em meio ácido com $3 \mathrm{~mol}$ $\mathrm{L}^{-1}$ de $\mathrm{H}_{2} \mathrm{SO}_{4}$; Fração 2 (F2) - diferença entre o $\mathrm{C}$ oxidado por $\mathrm{K}_{2} \mathrm{Cr}_{2} \mathrm{O}_{7}$ extraído em meio ácido com 6 e $3 \mathrm{~mol} \mathrm{~L}^{-1}$ de $\mathrm{H}_{2} \mathrm{SO}_{4}$; Fração 3 (F3) - diferença entre o $\mathrm{C}$ oxidado por $\mathrm{K}_{2} \mathrm{Cr}_{2} \mathrm{O}_{7}$ extraído em meio ácido com 9 e 6 mol L$^{-1}$ de $\mathrm{H}_{2} \mathrm{SO}_{4}$; e Fração 4 (F4) - diferença entre o $\mathrm{C}$ oxidado por $\mathrm{K}_{2} \mathrm{Cr}_{2} \mathrm{O}_{7}$ em meio ácido com $9 \mathrm{~mol} \mathrm{~L}^{-1}$ de $\mathrm{H}_{2} \mathrm{SO}_{4}$ e o COT.

Foram empregadas técnicas de análise multivariada como ferramenta estatística, especificamente a Análise de Componentes Principais (STATISTICA 7.0, 2004), para distinção do comportamento da matéria orgânica do solo nos diferentes solos sob Floresta Estacional Decidual.

\section{RESULTADOS E DISCUSSÃO}

Os maiores teores de COT foram observados nos horizontes com maiores teores de argila, principalmente no P1-NVe e P2-LVe, os quais se enquadram na classe textural argila-siltosa/muito argilosa e argila/muito argilosa, respectivamente (Tabela 2, 3). $A$ argila tem influência positiva na estabilização da matéria orgânica, uma vez que mudam o ambiente para os microrganismos, influenciam o $\mathrm{pH}$, força iônica, disponibilidade de substrato, produção e ati- vidade enzimática (Zech et al., 1997), além da proteção física promovida principalmente em microagregados.

O teor de COT variou entre os solos estudados, sendo os perfis de LVe (P2, P7 e P10) os que apresentaram maiores teores, com valores entre 33,7 e 58,2 $\mathrm{g} \mathrm{kg}^{-1}$ para o horizonte A, seguido do P1-NVe, com 46,1 $\mathrm{g} \mathrm{kg}^{-1}$, e em sequência o RLe, com $38,7 \mathrm{~g}$ $\mathrm{kg}^{-1}$ (Tabela 3). O teor de COT depende, essencialmente, do aporte de $\mathrm{C}$ e do processo de decomposição/mineralização da MOS. Em todos os perfis estudados o comportamento decrescente do COT, em função da profundidade do solo, foi observado. Os perfis derivados de calcário apresentaram maior saturação por bases (Tabela 2) e maiores valores de COT (Tabela 3), podendo haver relação direta da matéria orgânica do solo como fonte de nutrientes para o solo e a composição química da rocha.

Em relação às frações oxidáveis do COT (Tabela 3), de maneira geral, as frações F1 e F2 representaram a maior parte do COT para todos os horizontes estudados. Esse comportamento demonstra que a maior parte do carbono está na forma biodisponível (carbono lábil), sendo composto do aporte dos resíduos vegetais provenientes da cobertura vegetal, uma vez que as Florestas Estacionais Deciduais sazonalmente formam uma camada de serrapilheira. As frações F1 e F2 estão associadas com a disponibilidade de nutrientes e a formação de macroagregados (BLAIR ET al., 1995, CHAN ET Al., 2001), sendo a F1 a de maior labilidade no solo e altamente correlacionada com a fração leve livre da MOS (MAIA et al., 2007) 
Observando o comportamento de cada fração nota-se uma tendência dos horizontes superficiais apresentarem maior teor de $\mathrm{C}$ nas frações F1 e F2, o que pode ser justificado pelo contato direto com a serrapilheira, uma vez que esta é a principal fonte de biomassa nos ambientes nativos sob Floresta Estacional Decidual. Ocorre uma inversão deste comportamento para o $\mathrm{C}$ das frações $\mathrm{F} 3$ e F4, apresentando maiores valores no horizonte subsuperficial para a maioria dos perfis estudados. A relação $(\mathrm{F} 1+\mathrm{F} 2) /$
(F3+F4) apresentada na Tabela 3 é alta no horizonte superficial, indicando predomínio das frações mais lábeis (F1 e F2) e baixos no horizonte subsuperficial, indicando maior presença das frações mais recalcitrantes (F3 e F4) para a maioria dos perfis. A recalcitrância destas frações pode ser a explicação para seu maior acúmulo em subsuperfície, já que a precipitação na região é baixa, não permitindo a lixiviação total da fração, além da sua resistência a decomposição.

Tabela 2. Características morfológicas, químicas e físicas dos horizontes estudados

\begin{tabular}{|c|c|c|c|c|c|c|c|c|c|c|c|}
\hline Perfil & Hor. & Prof. & Litologia & $\mathrm{pH}$ & $S$ & $\mathrm{H}+\mathrm{Al}$ & $\mathrm{T}$ & $\mathrm{V}$ & Areia & Silte & Argila \\
\hline & & $\mathrm{cm}$ & & & 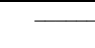 & $\mathrm{cmol}_{\mathrm{c}} \mathrm{kg}$ & $\bar{z}$ & $\%$ & 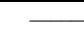 & $\mathrm{g} \mathrm{kg}^{-1}$ & $\bar{z}$ \\
\hline \multirow[t]{2}{*}{$\mathrm{P} 1$ - NVe } & A & $0-4$ & Calcário- & 6,84 & 13,80 & 2,0 & 15,8 & 87,3 & 90 & 410 & 500 \\
\hline & B2 & $22-53$ & Pelíticas & 6,19 & 8,62 & 3,0 & 11,62 & 74,2 & 70 & 220 & 710 \\
\hline \multirow[t]{2}{*}{ P2 - LVe } & A1 & $0-7$ & Calcário- & 7,01 & 14,38 & 2,0 & 16,38 & 87,8 & 380 & 200 & 420 \\
\hline & Bw1 & $31-77$ & Pelíticas & 6,01 & 4,79 & 2,2 & 6,99 & 68,5 & 330 & 40 & 630 \\
\hline \multirow[t]{2}{*}{ P3 - CXbe } & $\mathrm{AB}$ & $0-23$ & Calcário- & 7,18 & 13,89 & 1,4 & 15,29 & 90,8 & 120 & 360 & 520 \\
\hline & $\mathrm{Bi}$ & $51-78$ & Colúvio & 7,54 & 12,64 & 0,8 & 13,44 & 94,0 & 80 & 340 & 580 \\
\hline \multirow[t]{2}{*}{ P4 - RLe } & A & $0-10$ & & 6,51 & 14,79 & 1,9 & 16,69 & 88,6 & 530 & 230 & 240 \\
\hline & $\mathrm{C}$ & $10-30$ & & 6,29 & 9,58 & 2,0 & 11,58 & 82,7 & 520 & 240 & 240 \\
\hline \multirow[t]{2}{*}{ P5 - CXve } & A & $0-15$ & & 6,55 & 7,00 & 1,5 & 8,50 & 82,4 & 690 & 170 & 140 \\
\hline & $\mathrm{Bi}$ & $15-45$ & Granito & 5,96 & 2,74 & 1,7 & 4,44 & 61,7 & 690 & 210 & 100 \\
\hline \multirow[t]{2}{*}{ P6 - CXve } & A & $0-10$ & & 6,96 & 11,47 & 1,7 & 13,17 & 87,1 & 600 & 220 & 180 \\
\hline & $\mathrm{Bi}$ & $10-35$ & Granito & 6,25 & 6,56 & 2,4 & 8,96 & 73,2 & 560 & 230 & 210 \\
\hline \multirow[t]{2}{*}{ P7 - LVe } & A & $0-10$ & & 6,26 & 9,98 & 3,4 & 13,38 & 74,6 & 570 & 170 & 260 \\
\hline & Bwl & $20-70$ & Callatio & 5,27 & 5,62 & 1,9 & 7,52 & 74,7 & 520 & 160 & 320 \\
\hline \multirow[t]{2}{*}{ P8 -CXbd } & A & $0-10$ & Xisto- & 6,04 & 2,84 & 1,7 & 4,54 & 62,6 & 580 & 260 & 160 \\
\hline & $\mathrm{Bi}$ & $10-40$ & Verde & 4,96 & 0,94 & 2,1 & 3,34 & 28,1 & 580 & 230 & 190 \\
\hline \multirow[t]{2}{*}{ P9-CXbe } & A & $0-15$ & Colúvio- & 6,23 & 6,15 & 2,9 & 9,05 & 68,0 & 520 & 190 & 290 \\
\hline & $\mathrm{Bi}$ & $35-80$ & xistos & 6,22 & 3,87 & 2,0 & 5,87 & 65,9 & 350 & 190 & 460 \\
\hline \multirow[t]{2}{*}{ P10 - LVe } & A & $0-10$ & Granito- & 6,70 & 5,55 & 2,0 & 7,55 & 73,5 & 580 & 200 & 220 \\
\hline & Bw1 & $10-20$ & Gnaisse & 6,44 & 6,84 & 1,4 & 8,24 & 83,0 & 410 & 200 & 390 \\
\hline
\end{tabular}

pH: potencial hidrogeniônico medido em água (1:2,5); S: Soma de bases; $\mathrm{H}+\mathrm{Al}$ : acidez potencial; T: capacidade de troca de cátions potencial; e V: saturação por bases. 
Tabela 3. Teor de carbono orgânico total (COT) e frações de carbono oxidáveis em classes de solos de áreas de Floresta Estacional Decidual.

\begin{tabular}{|c|c|c|c|c|c|c|c|}
\hline \multirow[b]{2}{*}{ Perfil } & \multirow[b]{2}{*}{ Hor./Prof. (cm) } & COT & F1 & F2 & F3 & $\mathrm{F} 4$ & $\mathrm{~F} 1+\mathrm{F} 2 /$ \\
\hline & & & \multicolumn{4}{|c|}{$-\mathrm{g} \mathrm{kg}^{-1}$} & $\mathrm{~F} 3+\mathrm{F} 4$ \\
\hline \multirow[t]{2}{*}{$\mathrm{P} 1-\mathrm{NVe}$} & A $(0-4)$ & 46,1 & $14,1(30,6)$ & $17,4(37,8)$ & $5,5(11,9)$ & $9,1(19,7)$ & 2,2 \\
\hline & B2 (22-53) & 12,2 & $2,5(20,3)$ & $2,3(19,0)$ & $2,4(19,7)$ & $5,0(40,8)$ & 0,6 \\
\hline \multirow[t]{2}{*}{$\mathrm{P} 2-\mathrm{LVe}$} & A1 (0-7) & 58,2 & $16,2(27,9)$ & $13,6(23,4)$ & $16,5(28,4)$ & $11,8(20,3)$ & 1,1 \\
\hline & Bw1 (31-77) & 8,5 & $1,3(15,1)$ & $2,5(29,3)$ & $2,0(23,9)$ & $2,7(31,7)$ & 0,8 \\
\hline \multirow{2}{*}{ P3 - CXbe } & $\mathrm{AB}(0-23)$ & 38,4 & $7,8(20,3)$ & $9,0(23,5)$ & $10,0(25,9)$ & $11,6(30,2)$ & 0,8 \\
\hline & $\mathrm{Bi}(51-78)$ & 15,0 & $2,0(13,5)$ & $4,6(30,7)$ & $2,1(14,1)$ & $6,2(41,6)$ & 0,8 \\
\hline \multirow[t]{2}{*}{ P4 - RLe } & A $(0-10)$ & 38,7 & $9,2(23,8)$ & $7,2(18,5)$ & $9,9(25,5)$ & $12,5(32,3)$ & 0,7 \\
\hline & C (10-30) & 12,3 & $0,6(4,7)$ & $2,8(22,4)$ & $1,8(14,7)$ & $7,2(58,2)$ & 0,4 \\
\hline \multirow[t]{2}{*}{ P5 - CXve } & A $(0-15)$ & 23,7 & $6,0(25,3)$ & $4,6(19,3)$ & $6,6(27,9)$ & $6,6(27,7)$ & 0,8 \\
\hline & $\mathrm{Bi}(15-45)$ & 8,3 & $0,4(4,2)$ & $1,7(20,9)$ & $1,7(20,0)$ & $4,6(54,8)$ & 0,3 \\
\hline \multirow[t]{2}{*}{ P6 - CXve } & A $(0-10)$ & 27,5 & $8,5(30,9)$ & $5,7(20,7)$ & $11,2(40,8)$ & $2,1(7,6)$ & 1,1 \\
\hline & $\mathrm{Bi}(10-35)$ & 11,7 & $1,7(14,7)$ & $3,3(28,0)$ & $3,2(27,6)$ & $3,5(29,7)$ & 0,7 \\
\hline \multirow[t]{2}{*}{ P7 - LVe } & $\mathrm{A}(0-10)$ & 33,7 & $7,7(22,9)$ & $7,3(21,8)$ & $11,8(35,1)$ & $6,8(20,2)$ & 0,8 \\
\hline & Bw1 (20-70) & 6,5 & $1,3(20,7)$ & $1,3(19,6)$ & $1,4(22,3)$ & $2,4(37,1)$ & 0,7 \\
\hline \multirow[t]{2}{*}{ P8 - CXbd } & A $(10-35)$ & 11,5 & $3,1(26,7)$ & $1,6(14,0)$ & $4,6(40,0)$ & $2,2(19,3)$ & 0,7 \\
\hline & $\mathrm{Bi}(10-40)$ & 5,6 & $0,5(9,3)$ & $1,2(21,7)$ & $2,1(38,1)$ & $1,7(30,5)$ & 0,5 \\
\hline \multirow[t]{2}{*}{ P9 - CXbe } & A $(0-15)$ & 18,2 & $5,1(28,0)$ & $0,00(0,0)$ & $10,3(56,6)$ & $3,1(17,0)$ & 0,4 \\
\hline & $\mathrm{Bi}(35-80)$ & 7,6 & $1,3(17,3)$ & $2,4(31,8)$ & $1,6(20,7)$ & $2,3(30,2)$ & 1,0 \\
\hline \multirow[t]{2}{*}{ P10 - LVe } & $\mathrm{A}(0-10)$ & 35,0 & $7,6(21,8)$ & $5,5(15,8)$ & $11,6(33,2)$ & $10,2(29,1)$ & 0,6 \\
\hline & Bw2 (10-20) & 6,2 & $0,2(3,9)$ & $1,1(17,4)$ & $2,8(44,4)$ & $2,1(34,3)$ & 0,3 \\
\hline
\end{tabular}

F1 a F4: Frações oxidáveis de $\mathrm{C}$ orgânico em valores absolutos $\left(\mathrm{g} \mathrm{kg}^{-1}\right)$ obtidas por diferentes concentrações de $\mathrm{H}_{2} \mathrm{SO}_{4}$. Conc. $\mathrm{F} 1=3 \mathrm{~mol} \mathrm{~L}^{-1} \mathrm{H}_{2} \mathrm{SO}_{4}$; Conc. $\mathrm{F} 2=6-3 \mathrm{~mol} \mathrm{~L}^{-1} \mathrm{H}_{2} \mathrm{SO}_{4}$; Conc. F3 = 9-6 mol L-1 $\mathrm{H}_{2} \mathrm{SO}_{4}$; Conc. $\mathrm{F} 4=\mathrm{COT}-9 \mathrm{~mol} \mathrm{~L}^{-1}$; e valores entre parênteses são porcentagem das frações em relação ao COT.

Os teores de $\mathrm{C}$ das frações húmicas também apresentaram ampla variação entre os horizontes estudados, com C-FAF variando entre 0,6 e 3,67 g $\mathrm{kg}^{-1}$, C-FAH de 0,3 a $5,8 \mathrm{~g} \mathrm{~kg}^{-1}$ e o C-HUM de 3,0 a $40,8 \mathrm{~g} \mathrm{~kg}^{-1}$, sendo esta última a fração predominante, correspondendo em média a $65 \%$ do COT (Tabela 4). As relações C-FAH/C-FAF foram superiores a 1 para a maioria dos horizontes, denotando o predomínio dos ácidos húmicos nos diferentes tipos de solo sob Floresta Estacional Decidual. Essa relação pode indicar maior conversão do carbono orgânico insolúvel presente no solo em frações solúveis (MARTINS et al., 2009), entendendo-se que esta conversão é influenciada por outros fatores edáficos, como o $\mathrm{pH}$ do solo e o teor de argila, por exemplos. Em geral, os solos mais arenosos apresentam valores superiores para a relação, o que significa a perda seletiva da fração mais solúvel (FAF). Para a relação C-EA/CHUM (quociente entre o extrato alcalino $(\mathrm{EA}=\mathrm{C}$ $\mathrm{FAF}+\mathrm{C}-\mathrm{FAH}$ ) e o C-HUM) foram observados valores baixos, não superando 0,4 em nenhum horizonte. Essa relação entre o extrato alcalino e a fração humina pode ser indicativo de movimentação e acúmulo de $\mathrm{C}$ quando apresenta valores altos 
(SCHAEFER et al., 2002; Benites et al., 2001, 2003), porém este comportamento é típico de horizontes espódicos, denotando o processo de podzolização intenso.

Os baixos valores da relação C-EA/C-HUM indicam a alta estabilidade entre a matéria orgânica e a matriz mineral destes solos, proporcionada pela interação entre as argilas 2:1 e os íons $\mathrm{Ca}$ e/ou $\mathrm{Mg}$ com os grupos funcionais $\mathrm{COOH}$ e $\mathrm{OH}$ ionizados, principalmente da matéria orgânica (CANELLAS et al., 2008), explicando a alta correlação entre a saturação por bases e o teor de COT comentada anteriormente. A relação C-EA/COT foi próxima de zero, mostrando que as frações relacionadas aos ácidos fúlvicos e húmicos representam pouco o carbono orgânico existente ao longo dos perfis. A ampla variação dos valores dessas relações é um indicativo da heterogeneidade entre os materiais orgânicos, material de origem dos solos, bem como da diferença de intensidade do processo de humificação da matéria orgânica (FONTANA et al, 2010).

As porcentagens de cada fração em relação ao
COT (FAF, FAH e HUM) mostram o predomínio da fração humina, seguido dos ácidos húmicos (Tabela 4). Canellas et al. (2003) comentam que a presença da fração ácido húmico tem sido relacionada a solos férteis, com alto conteúdo de bases trocáveis. Observando as características químicas dos perfis estudados (Tabela 2) percebe-se que grande parte dos solos são eutróficos, com saturação por bases ultrapassando $60 \%$, o que relaciona positivamente a fertilidade do solo ao teor de ácidos húmicos. A ampla variação dos valores dessas relações anteriormente citadas é um indicativo da heterogeneidade entre os materiais orgânicos, material de origem desses solos, bem como da diferença de intensidade do processo de humificação da matéria orgânica. Como as florestas decíduas periodicamente perdem as folhas a formação de serapilheira e consequente acúmulo de biomassa é um processo contínuo, porém dependente da sazonalidade climática. Dessa forma, a variação da precipitação pode influenciar o acúmulo da MOS e os teores das frações húmicas presentes

Tabela 4. Distribuição do carbono orgânico das frações húmicas e relações nos horizontes estudados

\begin{tabular}{|c|c|c|c|c|c|c|c|c|c|c|c|}
\hline Perfil & Hor. & C-FAF & C-FAH & C-HUM & FAF & FAH & HUM & $\mathrm{EA}^{1}$ & $\begin{array}{l}\text { C-FAH/ } \\
\text { C-FAF }\end{array}$ & $\begin{array}{c}\text { C-EA/ } \\
\text { C-HUM }\end{array}$ & $\begin{array}{l}\text { C-EA } \\
\text { COT }\end{array}$ \\
\hline & & & $-\mathrm{g} \mathrm{kg}^{-1}$ & & 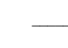 & -0 &  & - & & & \\
\hline \multirow{2}{*}{ P1 - NVe } & A & 2,50 & 5,80 & 35,9 & 5,30 & 12,6 & 78,0 & 17,9 & 2,4 & 0,2 & 0,1 \\
\hline & $\mathrm{B}_{2}$ & 0,70 & 1,30 & 8,80 & 5,50 & 10,4 & 72,2 & 15,9 & 1,9 & 0,2 & 0,2 \\
\hline \multirow{2}{*}{ P2 - LVe } & $\mathrm{A}_{1}$ & 3,60 & 4,90 & 40,8 & 6,20 & 8,50 & 70,1 & 14,7 & 1,4 & 0,2 & 0,1 \\
\hline & $\mathrm{Bw}_{1}$ & 0,60 & 1,20 & 6,30 & 7,10 & 14,1 & 74,1 & 21,2 & 2,0 & 0,3 & 0,2 \\
\hline \multirow{2}{*}{$\begin{array}{c}\text { P3 - } \\
\text { CXve }\end{array}$} & $\mathrm{AB}$ & 1,80 & 2,40 & 29,9 & 4,60 & 6,40 & 77,8 & 11,0 & 1,4 & 0,1 & 0,1 \\
\hline & $\mathrm{Bi}$ & 1,80 & 0,50 & 12,6 & 11,8 & 3,50 & 84,3 & 15,2 & 0,3 & 0,2 & 0,2 \\
\hline \multirow{2}{*}{ P4 - RLe } & A & 1,90 & 3,40 & 29,4 & 4,80 & 8,80 & 75,9 & 13,6 & 1,8 & 0,2 & 0,1 \\
\hline & $\mathrm{C}$ & 0,90 & 1,30 & 8,00 & 7,30 & 10,8 & 65,4 & 18,2 & 1,5 & 0,3 & 0,2 \\
\hline \multirow{2}{*}{$\begin{array}{c}\text { P5 - } \\
\text { CXve }\end{array}$} & A & 2,10 & 2,40 & 13,6 & 8,80 & 9,90 & 57,4 & 18,7 & 1,1 & 0,3 & 0,2 \\
\hline & $\mathrm{Bi}$ & 0,60 & 0,80 & 3,50 & 6,70 & 9,10 & 42,4 & 15,8 & 1,4 & 0,4 & 0,2 \\
\hline \multirow{2}{*}{$\begin{array}{c}\text { P6 - } \\
\text { CXve }\end{array}$} & A & 3,20 & 3,00 & 16,4 & 11,7 & 10,9 & 59,6 & 22,6 & 0,9 & 0,4 & 0,2 \\
\hline & $\mathrm{Bi}$ & 0,90 & 1,60 & 7,20 & 8,10 & 13,3 & 61,4 & 21,4 & 1,6 & 0,3 & 0,2 \\
\hline \multirow{2}{*}{ P7 - LVe } & A & 3,20 & 4,20 & 21,8 & 9,50 & 12,4 & 64,7 & 21,9 & 1,3 & 0,3 & 0,2 \\
\hline & $\mathrm{Bw}_{1}$ & 0,70 & 0,60 & 3,30 & 11,4 & 9,40 & 50,3 & 20,8 & 0,8 & 0,4 & 0,2 \\
\hline \multirow{2}{*}{$\begin{array}{c}\text { P8 - } \\
\text { CXbd }\end{array}$} & A & 1,00 & 1,20 & 6,80 & 9,00 & 10,4 & 58,8 & 19,4 & 1,1 & 0,3 & 0,2 \\
\hline & $\mathrm{Bi}$ & 0,60 & 0,30 & 3,00 & 10,3 & 4,80 & 54,0 & 15,2 & 0,5 & 0,3 & 0,2 \\
\hline \multirow{2}{*}{$\begin{array}{c}\text { P9 - } \\
\text { CXbe }\end{array}$} & A & 1,60 & 2,70 & 14,2 & 8,80 & 14,6 & 77,8 & 23,4 & 1,7 & 0,3 & 0,2 \\
\hline & $\mathrm{Bi}$ & 0,80 & 0,90 & 5,20 & 10,5 & 12,4 & 67,9 & 22,9 & 1,2 & 0,3 & 0,2 \\
\hline \multirow{2}{*}{$\begin{array}{l}\text { P10 - } \\
\text { LVe }\end{array}$} & A & 3,70 & 2,80 & 22,1 & 10,6 & 8,00 & 63,2 & 18,7 & 0,8 & 0,3 & 0,2 \\
\hline & $\mathrm{Bw}_{1}$ & 0,70 & 0,30 & 3,70 & 11,4 & 5,10 & 59,1 & 16,5 & 0,4 & 0,3 & 0,2 \\
\hline
\end{tabular}

C-FAF: carbono orgânico da fração ácidos fúlvicos; C-FAH: carbono orgânico da fração ácidos húmicos; C-HUM: carbono orgânico da fração humina; FAF; AH; HUM e EA (extrato alcalino): percentual de cada fração em relação ao carbono orgânico total. 
Ao observar o diagrama de ordenação dos componentes principais (Figura 3) percebe-se a distinção de dois grupos, sendo um formado pelos horizontes superficiais (A) de todos os perfis de solo avaliados nas áreas de Floresta Estacional Decidual e outro pelos horizontes subsuperficiais (B) destes mesmos perfis de solo. A formação de dois grupos bem distintos, com poucos pontos de sobreposição entre os casos avaliados, mostra que há uma forte distinção entre os horizontes quando se relacionam todos os atributos de solo estudados.

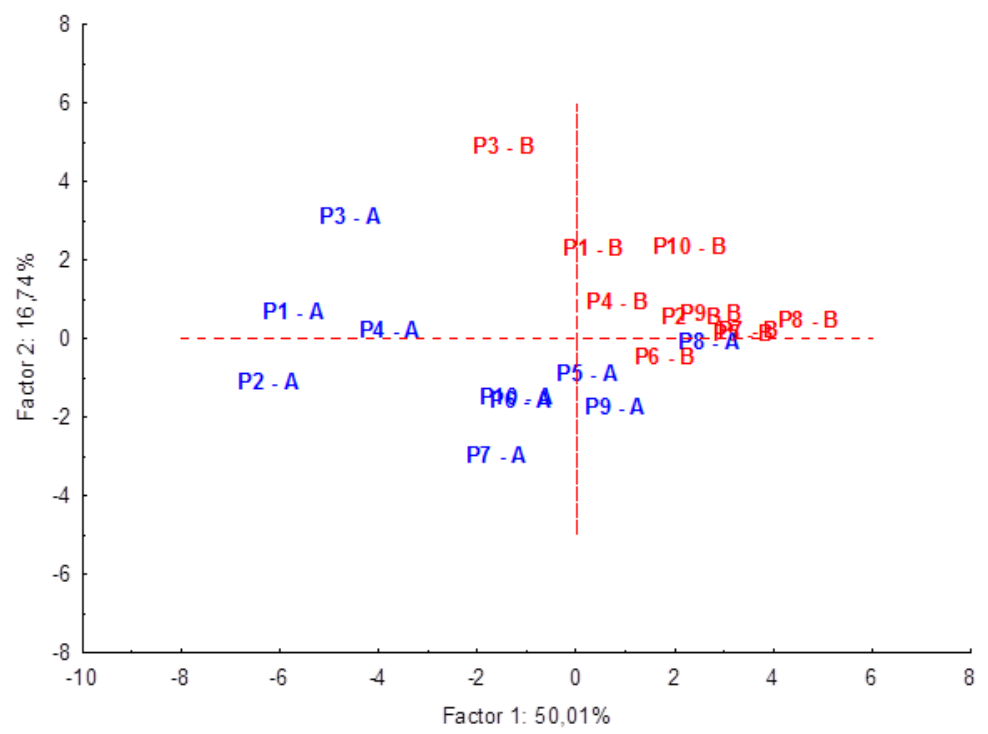

Figura 3. Diagrama de ordenação dos componentes principais formados a partir dos atributos avaliados nos horizontes superficiais (A) e subsuperficiais (B) dos perfis de solo sob Floresta Estacional Decidual.

Já no diagrama de projeção dos atributos de solo estudados nos perfis (Figura 4) percebe-se que os atributos que mais influenciaram para a distinção dos horizontes superficiais e subsuperficiais em dois grupos foram os atributos químicos, quais sejam, $\mathrm{pH}$, soma de bases, capacidade de troca catiônica e saturação por bases. Quanto aos atributos físicos apenas a fração areia influenciou na distinção dos dois gru- pos. Observando os diferentes compartimentos da matéria orgânica do solo avaliados nos horizontes fora examinado que praticamente todos os atributos relacionados (C-FAF, C-FAH, C-HUM, COT, F1, F2, F3 e F4) influenciaram na distinção dos grupos formados, apresentando elevados coeficientes de correlação, mostrando que apenas o Fator 1 explica $50,01 \%$ da variabilidade entre os dados (Tabela 5).

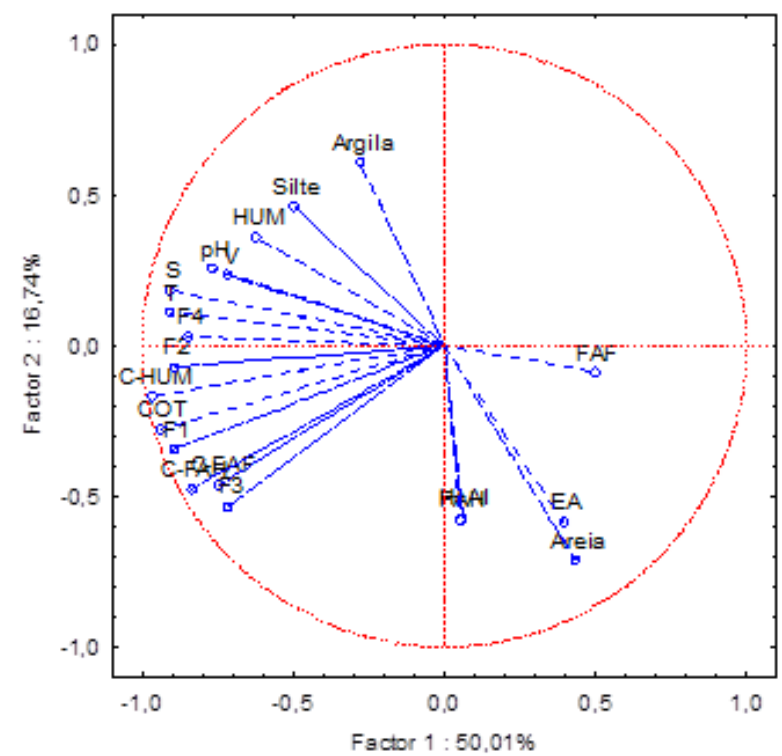

Figura 4. Diagrama de projeção dos atributos avaliados nos horizontes superficiais (A) e subsuperficiais (B) dos perfis de solo sob Floresta Estacional Decidual. S: soma de bases; V: saturação por bases; COT: carbono orgânico total; C-FAF: Teor de carbono na fração ácido fúlvico; C-FAH: Teor de carbono na fração ácido húmico; C-HUM: Teor de carbono na fração humina; FAF: porcentagem da fração ácido fúlvico em relação ao COT; FAH: porcentagem da fração ácido húmico em relação ao COT; HUM: porcentagem da fração humina em relação ao COT; e F1, F2, F3 e F4: Frações oxidáveis de carbono no solo. 
Percebe-se, com isso, a distinção dos compartimentos da matéria orgânica do solo em relação aos horizontes formados, mostrando que através dos referidos atributos pode-se interpretar o comportamento do carbono orgânico no solo e compreender sua influência no processo de pedogênese destes ambientes, uma vez que as Florestas Estacionais Deciduais apresentam grande aporte de material orgânico na superfície. Logo, os horizontes superficiais apresentam carbono orgânico do solo na forma mais lábil, estando prontamente disponível para os diversos processos bioquímicos envolvidos. Já os horizontes subsuperficiais apresentam carbono orgânico do solo na forma mais recalcitrante, sendo um importante aliado na estabilidade de agregados e consequente grau de desenvolvimento da estrutura do solo.

Tabela 5. Coeficientes de correlação dos componentes principais (Factor 1 e 2) dos atributos avaliados nos horizontes superficiais (A) e subsuperficiais (B) dos perfis de solo sob Floresta Estacional Decidual.

\begin{tabular}{|c|c|c|}
\hline \multirow[b]{2}{*}{ Atributos } & \multirow[b]{2}{*}{ Factor $1^{(1)}$} & \multirow[b]{2}{*}{ Factor $2^{(1)}$} \\
\hline & & \\
\hline $\mathrm{pH}$ & $-0,77$ & 0,26 \\
\hline $\mathrm{S}$ & $-0,91$ & 0,19 \\
\hline $\mathrm{H}+\mathrm{Al}$ & 0,06 & $-0,57$ \\
\hline $\mathrm{T}$ & $-0,91$ & 0,11 \\
\hline $\mathrm{V}$ & $-0,72$ & 0,23 \\
\hline Areia & 0,44 & $-0,71$ \\
\hline Silte & $-0,50$ & 0,46 \\
\hline Argila & $-0,28$ & 0,61 \\
\hline C-FAF & $-0,75$ & $-0,46$ \\
\hline C-FAH & $-0,84$ & $-0,48$ \\
\hline C-HUM & $-0,97$ & $-0,17$ \\
\hline FAF & 0,50 & $-0,09$ \\
\hline FAH & 0,05 & $-0,58$ \\
\hline HUM & $-0,62$ & 0,36 \\
\hline EA & 0,39 & $-0,58$ \\
\hline COT & $-0,94$ & $-0,28$ \\
\hline F1 & $-0,90$ & $-0,35$ \\
\hline $\mathrm{F} 2$ & $-0,90$ & $-0,07$ \\
\hline F3 & $-0,72$ & $-0,53$ \\
\hline F4 & $-\mathbf{0 , 8 5}$ & 0,03 \\
\hline Variância total (\%) & 50,01 & 16,74 \\
\hline Variância acumulada (\%) & 50,01 & 66,75 \\
\hline
\end{tabular}

\footnotetext{
${ }^{(1)}$ Fatores $\geq|0,70|$ são significativos (Manly, 1994).
} 


\section{CONCLUSÕES}

Os solos sob Floresta Estacional Decidual apresentaram a seguinte sequência de predominância das substâncias húmicas, quais sejam, Humina, Ácidos Húmicos, e Ácidos Fúlvicos, denotando forte relação entre a Humina e os perfis de solos mais argilosos, favorecendo maior formação de frações mais recalcitrantes e a presença maior dos ácidos húmicos relacionada ao caráter eutrófico da maioria dos perfis.

Quanto às frações de $\mathrm{C}$ oxidáveis, houve variação entre a fração de $\mathrm{C}$ mais lábil e mais recalcitrante, predominando as mais lábeis nos horizontes superficiais e as mais recalcitrantes em subsuperfície.

Foram formados dois grupos através da análise de componentes principais, distinguindo os horizontes superficiais dos subsuperficiais de todos os perfis estudados. Sendo os diferentes compartimentos da matéria orgânica do solo os principais influenciadores desta distinção.

\section{REFERÊNCIAS}

BENITES, V. M. et al. Caracterização da matéria orgânica e micromorfologia de solos sob campos de altitude no Parque Estadual da Serra do Brigadeiro MG. Revista Brasileira de Ciência do Solo, Viçosa, v. 25, n. 3, p. 661-674, 2001.

BENITES, V. M. et al. Solos e Vegetação nos Complexos Rupestres de Altitude da Mantiqueira e do Espinhaço. Revista Floresta e Ambiente, Seropédica, v. 10, n. 1, p. 76-85, 2003.

BENITES, V. M.; MADARI, B.; MACHADO, P. L. O. A. Extração e fracionamento quantitativo de substâncias húmicas do solo: Um procedimento simplificado de baixo custo. Rio de Janeiro: EMBRAPA SOLOS, 2003.7 p. (Comunicado Técnico, 16).

BLAIR, G. J.; LEFROY, R. D. B.; LISLE, L. Soil carbon fractions based on their degree of oxidation, and development of a carbon management index for agricultural systems. Australian Journal of Agricultural Research, Melboune, v. 46, n. 7, p. 14591466, 1995.

CANELLAS, L. P. et al. Propriedades químicas de um Cambissolo cultivado com cana-de-açúcar, com preservação do palhiço e adição de vinhaça por longo tempo. Revista Brasileira de Ciência do Solo, Viçosa, v. 27, n. 5, p. 935-944, 2003.

CANELLAS, L. P. et al. Reações da matéria orgânica. In: SANTOS, G. A. et al. (eds). Fundamentos da matéria orgânica do solo: Ecossistemas tropicais \& subtropicais. 2.ed. Porto Alegre, Metrópole, 2008. p.45-64.

CHAN, K. Y.; BOWMAN, A.; OATES, A. Oxidizidable organic carbon fractions and soil quality changes in an oxic paleustalf under different pasture leys. Soil Science, Philadelphia, v. 166, n. 1, p. 61 67, 2001.

EMPRESA BRASILEIRA DE PESQUISA AGROPECUÁRIA - EMBRAPA. Manual de métodos de análise de solo. 2 . ed. Rio de Janeiro, RJ: Centro Nacional de Pesquisa de Solos, 1997. 212p.

ESPÍRITO-SANTO, M. et al. Bases para a conservação e uso sustentável das florestas estacionais deciduais brasileiras: a necessidade de estudos multidisciplinares. Unimontes Científica, Montes Claros, v. 8, n. 1, p. 13-14, 2006.

FONTANA, A. et al. Quantificação e utilização das frações húmicas como característica diferencial em horizontes diagnósticos de solos brasileiros. Revista Brasileira de Ciência do Solo, Viçosa, v. 34, n. 4, p. 1241-1257, 2010.

JANZEN, D. H. Management of habitat fragments in a tropical dry forest: Growth. Annals of the Missouri Botanical Garden, St. Louis, v. 75, n. 1, p. 105-116, 1988

IBGE. Instituto Brasileiro de Geografia e Estatística. Mapa dos Biomas do Brasil - Primeira Aproximação. Escala 1:5.000.000. Brasília, DF. 2004.

INMET. Instituto Nacional de Meteorologia. Banco de Dados Meteorológicos para Ensino e Pesquisa. Disponível em http://www.inmet.gov.br/portal/ index.php? $\mathrm{r}=\mathrm{bdmep} / \mathrm{bdmep}$. Acesso em 01 de julho de 2015

MAIA, S. M. F. et al. Organic carbon pools in a Luvisol under agroforestry and conventional farming systems in the semi-arid region of Ceará, Brazil. Agroforestry Systems, Dordrecht, v. 71, n. 2, p.127138. 2007

MANLY, B. F. J. Multivariate statistical methods 2.ed. London, Chapman \& Hall, 1994. 215 p.

MARTINS, E. L.; CORINGA, J. E. S.; WEBER, O. L. S. Carbono orgânico nas frações granulométricas e substâncias húmicas de um Latossolo Vermelho Amarelo distrófico - LVAd sob diferentes agrossistemas. Acta Amazonica, Manaus, v. 39, n. 3, p. 655 $660,2009$.

MENDONÇA, E. S.; MATOS, E. S.. Matéria Orgânica do Solo: Métodos de Análises. Viçosa: UFV, 
2005. $107 \mathrm{p}$.

MIRANDA, C. C.; CANELlAS; L. P.; NASCIMENTO, M. T.. Caracterização da Matéria Orgânica do Solo em Fragmentos de Mata Atlântica e em Plantios Abandonados de Eucalipto. Revista Brasileira de Ciência do Solo, Viçosa, v. 31, n. 5, p. 905 916. 2007.

PRADO, D. E.; GIBBS, P. E. Patterns of species distributions in the dry seasonal forest South America. Annals of the Missouri Botanical Garden, St. Louis, v. 80, n. 1, p. 902-927, 1993.

SANTOS, R. D. et al. Manual de descrição e coleta de solo no campo. 6.ed. Viçosa: SBCS, 2013. 100p

SCARIOT, A.; SEVILHA, A. C. Biodiversidade, estrutura e conservação de florestas estacionais deciduais no Cerrado. In: SCARIOT, A.; SOUSASILVA, J. C.; FELFILI, J. M. (Ed.). Cerrado: Ecologia, Biodiversidade e Conservação. Brasília: Ministério do Meio Ambiente, 2005. cap. 6, p. 121139.

SCHAEFER, C. E. G. R. et al. Pedogenesis on the up lands of the Diamantina Plateau, Minas Gerais, Brazil: a chemical and micropedological study. Geoderma, Philadelphia, v. 107, n. 3, p.243-269. 2002.

SIQUEIRA, A. S.; ARAÚJO, G. M.; SCHIAVINI, I. Estrutura do componente arbóreo e características edáficas de dois fragmentos de floresta estacional decidual no vale do rio Araguari, MG, Brasil. Acta Botanica Brasilica, São Paulo, v. 23, n. 1, p. 10-21. 2009.

SWIFT, R. S. Organic matter characterization. In: SPARKS, O.L., (Ed.) Methods of soil analysis Part 3: Chemical methods. Madison: Soil Science Society of America, 1996. n. 5, cap. 35, p.1011-1020.

YEOMANS, J. C., BREMNER, J. M. A rapid and precise method for routine determination of carbon in soil. Communications in Soil Science and Plant Analysis, Nova Iorque, v. 19, n. 13, p. 1467-1476, 1988.

WERNECK, M. S.; FRANCESCHINELLI, E. V.; TAMEIRÃO-NETO, E. Mudanças na florística e estrutura de uma floresta decidual durante um período de quatro anos (1994-1998), na região do Triângulo Mineiro, MG. Revista Brasileira de Botânica, São Paulo, v. 23, n. 4, p. 401-413, 2000.

ZECH, W. et al. Factors controlling humification and mineralization of soil organic matter in the tropics. Geoderma. Philadelphia, v. 79, n.3, p. 117-161, 1997. 